\title{
A Short Review on Proteomics and its Applications
}

\author{
K. Chandrasekhar ${ }^{1}$, A. Dileep ${ }^{2}$, D. Ester Lebonah ${ }^{1}$, J. Pramoda Kumari ${ }^{1, *}$ \\ ${ }^{1}$ Research Scholar, Department of Microbiology, S.V. University, Tirupati, \\ Andhra Pradesh, India \\ *Fax No: +91-0877-2289555 \\ ${ }^{2}$ Junior Research Fellow, Department of Microbiology, S.V. University, Tirupati, \\ Andhra Pradesh, India \\ *E-mail address: prmodkumarij@gmail.com
}

\begin{abstract}
Proteomics is the large scale of study of proteins, particularly their function and structure. Proteomics is an excellent approach for studying changes in metabolism in response to different stress conditions. In the present review focused on different types of techniques for the analysis of expressed proteins. The techniques includes 2-D gel electrophoresis, MALDI-TOF/MS etc., play a vital role for the analysis of novel proteins and their role in disease maintenance and treatment. The review also concentrated on applicative perspective of proteomics in the fields of biomedical, agriculture and food.
\end{abstract}

Keywords: Proteomics; 2-D electrophoresis; MALDI-TOF/MS; Matrix; Applications

\section{INTRODUCTION}

Protein, highly complex substance that is present in all living organisms. Proteins are the polymers of amino acids. Emil Fischer and Franz Hofmeister, reported about proteins in 1902, Proteins play an important role in metabolic activities. Primary structure of protein is determined by the sequence of specific amino acids, encoded by the mRNA, which directs the proper folding of the polypeptide chain into the secondary structure. One type of secondary structure is the alpha helix, a region of the polypeptide that folds into a corkscrew shape. Beta strands are linear structures of polypeptides, bonding together to form a flat beta sheet. Turns and coils interact chemically with each other to form the unique three dimensional shape of the proper three dimensional structure creates the final protein. Many proteins, however, have several different polypeptide subunits that make the final active protein. For these proteins, the interactions between the different subunits form the quaternary structure. One of the most promising developments to come from the study of human genes and proteins has been the identification of potential new drugs for the treatments of disease. This relies on genome and proteome information to identify proteins associated with a disease. The term "proteomics" was first coined in 1995 and was defined as the large-scale characterization of the entire protein complement of a cell line, tissue, or organism $^{1,11,12}$. Proteomics is the large-scale study of proteins particularly their composition, structures, functions, and interactions of the proteins directing the activities of cell ${ }^{2,3}$. The 
main theme of interest proteomics it gives a much better understanding of an organism than genomics. Genomics can give a rough estimation of expression of a protein. Most of the proteins function in collaboration with other proteins, and the main goal of proteomics are to identify which proteins interact. After genomics, proteomics is often considered as the advanced step in the study of biological systems. It is much more complicated than genomics, mostly because while an organism's genome is more or less constant, the total protein expression profile always changes with time, micro and macro environmental conditions.

Mass spectrometry (MS) has been widely used in forensic science in the identification of compounds, particularly illicit drugs. MS is a technique that allows the detection of compounds by separating ions by their unique mass (mass-to-charge ratios) using a mass spectrometer. The method relies on the fact that every compound has a unique fragmentation pattern (mass spectrum). The sample is ionized; the sample ions are separated based on their differing masses and relative abundance.

\section{TYPES OF PROTEOMICS}

Based on the protein response under stress conditions proteomics are classified into different groups.

\section{1. Expression proteomics}

Expression proteomics is used to study the qualitative and quantitative expression of total proteins under two different conditions. Like the normal cell and treated or diseased cell can be compared to understand the protein that is responsible for the stress or diseased state or the protein that is expressed due to disease. Typically, expression proteomics studies are addressed to the investigation of the expression protein patterns in abnormal cells. Ex. Compare tumor tissue sample and the normal tissue can be analyzed for differential protein expression. 2-D gel electrophoresis, mass spectrometry technique were used to observed the protein expressional changes, which is present and absent in tumor tissue, when compared with normal tissue. Which are over expressed and under expressed can be identified and characterized protein activities multi-protein complexes, and signalling pathways ${ }^{4,5}$. Identification of these proteins will give valuable information about molecular biology of tumor formation and disease-specific manner for use as diagnostic markers or therapeutic targets ${ }^{6}$.

\section{2. Structural proteomics}

Structural proteomics helps to understand three dimensional shape and structural complexities of functional proteins. Structural prediction of a protein when its amino acid sequence is determined directly by sequencing or from the gene with a method called homology modelling. Structural proteomics can give detailed information about the structure and function of protein complexes present in a specific cellular organelle. It is possible to identify all the proteins present in a complex system such as membranes, ribosomes, and cell organelles and to characterise all the protein interactions that can be possible between these proteins and protein complexes. Different technologies such as X-ray crystallography and NMR spectroscopy were mainly used for structure determination ${ }^{7}$. 


\section{3. Functional proteomics}

Functional proteomics explains understanding the protein functions as well as unrevealing molecular mechanisms within the cell then depend on the identification of the interacting protein partners. The association of an unknown protein with partners belonging to a specific protein complex involved in a particular mechanism would in fact, be strongly suggestive of its biological function ${ }^{8,9}$. Furthermore detailed description of the cellular signalling pathways might greatly benefit from the elucidation of protein- protein interactions in-vivo ${ }^{10}$.

\section{4. Techniques involved in proteomics}

In proteomic analysis both analytical and bio-informatics tools were used to characterize protein structure and functions. Analytical techniques 2-D gel electrophoresis, MALDI-TOF-MS were used. In case of bio-informatics numbers of software tools were used.

\section{5. 2-D gel electrophoresis}

In 2-D gel electrophoresis, protein samples are resolved based on charge, in a step called isoelectric focusing, and then based on molecular weight in second step ${ }^{13}$. The result is an image in thousands of small spots, each representing a protein. A good 2-D gel can resolve one thousand to two thousand protein spots, which appear after staining, as dots in the gel. 2-D gel electrophoresis technique is mainly used to compare two similar samples to find specific protein differences.

\section{6. 2-D Electrophoresis workflow chart}

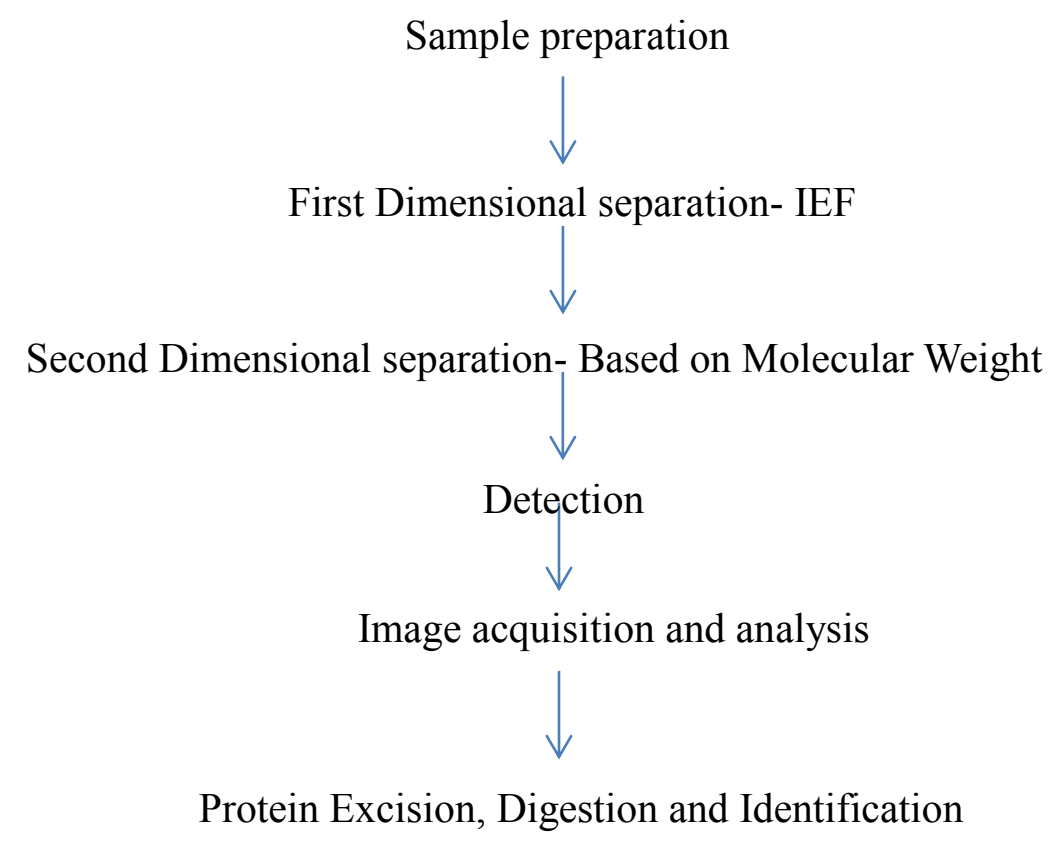

Fig. 1. 2-D Electrophoresis workflow chart.

Prepare the protein at a concentration and in a solution suitable for IEF. Choose a method that maintains the native charge, solubility, and relative abundance of proteins of interest. Separate proteins according to $\mathrm{pI}$ by IEF. Select the appropriate IPG strip length and 
$\mathrm{pH}$ gradient for the desired resolution and sample load. Select appropriate sample loading and separation conditions. Separate proteins according to size by SDS-PAGE. Select the appropriate gel size and composition and separation conditions. Visualize proteins using either a total protein stain or fluorescent protein tags. Select a staining technique that matches sensitivity requirements and available imaging equipment. Capture digital images of the 2-D patterns using appropriate imaging equipment and software. Then analyze the patterns using 2-D software. Excise protein spots of interest from the gel digest the proteins, and the digests by MS.

\section{7. MS analysis}

Mass spectrometry is an analytical technique that produces spectra of the masses of the atoms or molecules comprising a sample of material. The spectra are used to determine the elemental or isotopic signature of a sample, the masses of particles and of molecules, and to elucidate the chemical structures of molecules, such as peptides and other chemical compounds. Mass spectrometry works by ionizing chemical compounds to generate charged molecules or molecule fragments and measuring their mass to charge ratios ${ }^{14}$. MALDI-TOF is the most useful technique for protein identification.

\section{8. MALDI-TOF-MS}

Matrix Assisted Laser Desorption/Ionisation is a soft ionization technique used in spectrometry, allowing to analysis the biomolecules like DNA, protein, peptides. Biomolecules and synthetic polymers have low volatility and are thermally unstable, which has limited the use of MS as a means of characterization. These problems have been minimized through the development of MALDI-TOF MS, which allows for the mass determination of biomolecules by ionization and vaporization without degradation, a Laser beam used to ionize the sample ${ }^{15}$.

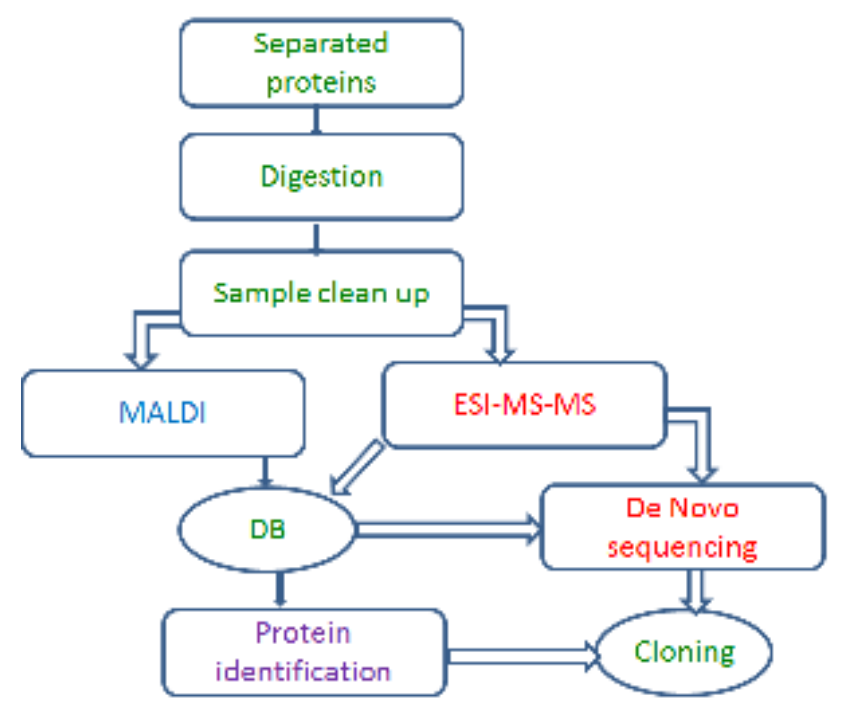

Fig. 2. MALDI-TOF -MS analysis representing image. 
Protein sample have been characterized by HPLC or SDS PAGE by generating peptide maps. These peptide maps have been used as fingerprints of protein or as a tool to know the purity of a known protein in a known sample. Mass spectrometry gives a peptide map when proteins are digested with proteolytic enzymes like trypsin. This peptide map can be used to search a sequence database to find a good match from the existing database.

\subsection{Sample preparation}

MALDI-TOF MS is used to characterise, biomolecules like proteins, peptides and polymers of organic compounds. Sample preparation for MALDI-TOF is very interesting and important step. Purify the protein sample before going MALDI-TOF analysis because it is more tolerant to sample contaminants but contaminants can seriously disturb incorporation of sample molecules with growing matrix crystals. Sample can mix with matrix in 1:2 ratio. Different types of matrices are used based on sample, some of matrix are 2-(4-hydroxy phenylazo benzoic acid, 2,4,6-trihydroxyacetophenone, 3-aminoquinolone, cinnamic acid, etc.). Dried droplet technique is predominantly applied for MALDI-TOF analysis, protein sample, mixed with matrix on a metal plate. The combination of matrices yielded slightly performance $^{16}$. Small volumes should be used for standard metal plates. On the other hand, hydrophilic sample anchors are efficient for the generation of small spots ${ }^{17}$.

\section{10. Matrix}

A good matrix consist the following properties i.e. Matrix must be able to absorb U.V wavelength of usually $237 \mathrm{~nm}$, being easily exited and ability to transfer of proton to the sample molecules. Main role of the matrix is adsorption of energy from laser pulse, and then transfer to sample this energy can causes the vaporisation of the sample. For protein samples typical MALDI matrix consist of hydroxylated benzoic acid and cinnamic acid derivatives.

\section{ADVANCED METHODS IN PROTEOMICS}

\section{1. Isotope-coded affinity tags (ICAT)}

It is a gel- free method for quantitative proteomics that relies on chemical labelling reagents. These chemical probes consist of three general elements i.e. defined amino acid side chain, an isotopically coded linker, and a tag for the affinity isolation of labelled proteins/peptides. For quantitative comparison of two proteomes, sample labelled with isotopically light, and other one is heavy version. Both samples combined with isotope-coded tagging reagents. These peptides are analyzed by LC-MS. Tags were deuterium, ${ }^{13} \mathrm{C}{ }^{18}$. The technique mainly used the relative quantification of proteins present in two or more biological samples. Visible isotope-coded affinity tags are the additional method in ICATVisible tag that allows the electrophoresis position of tagged peptides to be easily monitored.

\section{2. Isobaric Tags for Relative and Absolute Quantification (iTRAQ)}

Isobaric tags for relative and absolute quantitation (iTRAQ), it is also a non- gel- based technique used to quantify proteins. iTRAQ is used in proteomics to study quantitative changes in the proteome ${ }^{19}$. Based on the covalent labelling of the $\mathrm{N}$-terminus and side chain amines of peptides from protein digestions with tags of varying mass, 4-plex and 8-plex are the reagents can be used to label all peptides from different samples. The samples can be 
analysed by using mass spectrometry MS/MS. Different types of software's are available for analyse the MS/MS spectra's i.e. j-Tracker, j-TraqX ${ }^{20}$.

\section{3. Absolute Quantification (AQUA)}

AQUA, studies the absolute quantification of proteins and their modification sates. Covalent modifications can be used to prepare synthetic proteins. These modifications are chemically identical to naturally occurring posttranslational modifications. These types of peptides used to quantify the post translational modified proteins after proteolysis with the help of tandem mass spectrometer.

\section{4. ESI-Q-IT-MS}

Micro electrospray ionization (ESI)-Quadrupole ion trap (QIT) Time of flight (TOF) mass spectrometer (MS) has a very good resolution. In ESI ionisation proteins are ionised in solution and carry multiple charge state. The advantage of using ESI-QTOF analysis for protein mass determination is that due to the high charge state of proteins their $\mathrm{m} / \mathrm{z}$ measurements is typically less than 2000 and the TOF detector has a very good mass accuracy in this scan range. This result is more accurate mass measurements for proteins in ESI-QTOF.

\section{5. SELDI-TOF-MS}

The technique Surface-enhanced laser desorption/ionization (SELDI) is used for the analysis of protein mixtures, it is an ionization method in mass spectrometry ${ }^{21}$. SELDI is typically used with time-of-flight mass spectrometers and is used to detect proteins in clinical samples; to compare protein levels with and without a disease can be used for biomarker discovery $^{22}$.

\section{6. Applications of proteomics}

Proteomics is widely used technique in biological fields, mainly applied in Oncology (Tumor biology), Bio-medicine, Agriculture and Food Microbiology.

\section{7. Oncology}

Oncology refers study of Tumor cell, Tumor metastasis, is the process spread of cancer from one organ to another non-adjacent organ cause death in patients ${ }^{24}$. The major challenge in medicine to describe the molecular and cellular mechanisms underlying tumor metastasis. Analyse the protein expressions correlated to the metastatic process which help to understand the mechanism of metastasis and thus facilitate the development of strategies for the therapeutic interventions and clinical management of cancer. Proteomics is a systematic research, the main aim of this research is to characterize the protein expressions, functions of tumor cells and widely used in biomarker discovery.

\section{8. Bio-medical applications}

The study of interactions between microbial pathogens and their hosts is called "infectomics". It is very interesting area in proteomics. It deals with the fundamentals of the infections origin and their effect on organs. The main aim of this research is to prevent or cure disease at starting level. Advanced diagnostic issues related to emerging infections, increasing of fastidious bacteria, and generation of patient- tailored phenotypes ${ }^{24}$. 


\section{9. Agricultural applications}

The applications of plant proteomics scientific research is still in budding stage. Proteomics is also used to know plant-insect interactions that help identify candidate genes involved in the defensive response of plants to herbivore ${ }^{23}$. Population growth and effect of global climate changes imposing severe limits on the sustainability of agricultural crop production.

\section{10. Food Microbiology}

The use of proteomics in food technology is presented especially for characterisation and standardisation of raw materials, process development, and detection of batch-to batch variations and quality control of the final product. Further attention is paid to the aspects of food safety, especially regarding biological and microbial safety and the use of genetically modified foods ${ }^{25}$.

\section{CONCLUSION}

Based on the above findings the present review was concluded that the applications for proteomics are relevant to all of the biological process and provides a means to utilise the expressed protein data in a more effective way.

\section{References}

[1] Anderson N.G., Anderson N.L., Electrophoresis 17 (1996) 443-453.

[2] Anderson N.L., Anderson N.G., Electrophoresis 19( 1998) 1853-1861.

[3] Blackstock W.P., Weir M.P., Treands in Biotechnology 17(1999) 121-127.

[4] Pandey A., Fernandez M.M., Stehen H., Blagoev B., Nielsen M.M., Roche S., Mann M., Lodish H.F., Journal of Biological Chemistry 275(2000) 38633-38639.

[5] Hinsby A.M., Olsen J.V., Bennett K.L., Mann M., Molecular and Cell Proteomics 2 (2003) 29-36.

[6] Banks R.E., Dunn M.J., Hochstrasser D.F., Sanchez J.C., Blackstock W., Pappin D.J., Selby P.J., Lancet 356 (2000) 1749-1756.

[7] Yokoyama S., Hirota H., Kigawa T., Yabuki T., Shirouzu M., Terada T., Ito Y., Massuo Y., Kuroda Y., Nishimura Y., Kyogoku Y., Miki K., Masui R., Kuramitsu S., Nature Structural and Molecular Biology 7 (2000) 943-945.

[8] Gavin A.C., Bosche M., Krause R., Grandi P., Marzioch M., Bauer A., Nature 415 (2002) 141-147.

[9] Ho Y., Gruhler A., Heilbut A., Bader G.D., Moore L., Adams S.L., Millar A., Taylor P., Bennett K., Boutilier K., Yang L., Wolting C., Donaldson I., Schadoeff S., Nature 415 (2002) 180-183.

[10] Lewis T.S., Hunt J.B., Aveline L.D., Jonscher K.R., Louie D.F., Yeh J.M., Nahreini T.S., Resing K.A., Ahn N.G., Molecular Cell. 6 (2000) 1343-1354. 
[11] Wasinger V.C., Cordwell S.J., Cerpa-Poljak A., Yan J.X., Gooley A.A., Wilkins M.R., Duncan M.W., Harris R., Williams K.L., Humphery- Smith I., Electrophoresis 16 (1995) 1090-1094.

[12] Wilkins M.R., Sanchez J.C., Gooley A.A., Appel R.D., Humphery- Smith I., Hochstrasser D.F., Williams K.L., Biotechnology and Genetic Engineering Reviews 13 (1995) 19-50.

[13] Mikkelsen Susan, Cortón Eduardo, Bioanalytical Chemistry. John Wiley \& Sons, Inc. p. 224 (2004).

[14] Sparkman David O., Mass spectrometry desk reference. Pittsburgh: Global View Pub. 2000.

[15] Wu K.J., Shaler T.A., Becker C.H., Analytical Chemistry 66 (1994) 1637-1645.

[16] Bak-Jensen K.S., Laugesen S., Roepstorff P., Svensson B., Proteomics 4 (2004) 992-1002.

[17] Schuerenberg M., Luebbert C., Eickho V.H., Kalkum M., Lehrach E.H., Nordho V., Analytical Chemistry 72(2000) 3436-3442.

[18] Yi E.C., Li X.J., Cooke K., Lee H., Raught B., Page A., Aneliunas V., Hieter P., Goodlett D.R., Aebersold R., Proteomics 5 (2005) 380-387.

[19] Zieske L.R., Journal of Experimental Botany 57(2006) 1501-1508.

[20] Muth T., keller D., Puetz S.M., Maetens L., Sickmann A., Boehm A.M., Proteomics 10 (2010) 1223-1225.

[21] Tang N., Tornatore P., Weinberger S.R., Mass spectrometry reviews 23(2004) 34-44.

[22] Li J., Zhang Z., Rosenzweig J., Wang Y.Y., Chan D.W., Clinical Chemistry 48 (2002) 1296-1304.

[23] Sangha J.S., Chen Y.H., Kaur J., Khan Wajahatullah, Abduljaleel Zainularifeen, Alanazi Mohammaed S., Mills Aaron., Adalla C.B., Bennett John., Balakrishnan P., Jahn G.C., Hei Leung., International Journal of Molecular Sciences 14 (2013) 3921-3945.

[24] Chiang A.C., Massague J., The new England Journal of Medicine 359 (2008) 2814-2823.

[25] Dajana Gaso Sokac, Spomenka Kova, Djuro Josi, Food Technology and Biotechnology 48 (2010) 284-295.

[26] Del Chierico F., Petrucca A., Vernocchi P., Bracaglia G., Fiscarelli E., Bernaschi P., Muraca M., Urbani A., Putignana L., Journal of proteomics 97 (2014) 69-87. 